Research Article

\title{
Coexistence of Two Cubic-Lattice Co Matrices at High Temperatures in Co-Re-Cr-Ni Alloy Studied by Neutron Diffraction
}

\author{
Přemysl Beran, ${ }^{1}$ Debashis Mukherji $(\mathbb{D}),{ }^{2}$ Pavel Strunz $(\mathbb{D}),{ }^{1}$ Ralph Gilles $\left(\mathbb{D},{ }^{3}\right.$ Markus Hölzel, ${ }^{3}$ \\ and Joachim Rösler ${ }^{2}$ \\ ${ }^{1}$ Nuclear Physics Institute of the CAS, Řež 130, 25068 Řzz, Czech Republic \\ ${ }^{2}$ Institut für Werkstoffe, Technische Universität Braunschweig, Langer Kamp 8, 38106 Braunschweig, Germany \\ ${ }^{3}$ Heinz Maier-Leibnitz Zentrum (MLZ), Technische Universität München, Lichtenbergstr 1, 85747 Garching, Germany
}

Correspondence should be addressed to Pavel Strunz; strunz@ujf.cas.cz

Received 28 February 2018; Accepted 17 April 2018; Published 17 May 2018

Academic Editor: Frederic Dumur

Copyright (C) 2018 Přemysl Beran et al. This is an open access article distributed under the Creative Commons Attribution License, which permits unrestricted use, distribution, and reproduction in any medium, provided the original work is properly cited.

In situ neutron diffraction measurements were performed during heating to high temperature and cooling for a Co-17Re-23Cr$25 \mathrm{Ni}$ alloy. The allotropic transformation of the Co matrix and the evolution of the low-temperature hexagonal and hightemperature cubic Co phases were studied. A surprising observation was the splitting of the face-centred cubic (fcc) Co phase peaks at high temperature during heating as well as cooling. The phase evolution was monitored, and an appearance of the secondary fcc phase could be linked to the formation of $\sigma$ phase $\left(\mathrm{Cr}_{2} \mathrm{Re}_{3}\right.$ type $)$ associated with a compositional change in the matrix due to diffusion processes at high temperature.

\section{Introduction}

Amongst new alloy systems being developed to supplement $\mathrm{Ni}$-based superalloys in gas turbine application, the Co-Rebased alloys show promise because of their excellent specific strength and relatively high melting range $\left(1490^{\circ} \mathrm{C}-1560^{\circ} \mathrm{C}\right)$ [1]. Like other Co-based superalloys presently used in gas turbine static components, the Co-Re alloys are also strengthened by monocarbide $\mathrm{TaC}$ [2] and use $\mathrm{Cr}$ to provide oxidation resistance [3]. The Co-Re alloys designed at TU Braunschweig are now being investigated for improving oxidation resistance and simultaneously to partially replace $\mathrm{Cr}$ by addition of $\mathrm{Ni}$. Cr addition above $20 \%$ (all composition in this paper are in atomic percentage) pose two challenges-namely, the formation of topologically closedpacked $\mathrm{Cr}_{2} \mathrm{Re}_{3}$-type $\sigma$ phase and the partial stabilisation of $\mathrm{Cr}$-carbides that are unstable above $1000^{\circ} \mathrm{C}$ [4]. The different non-nickel-containing Co-Re alloy compositions studied so far [5] showed that the matrix in these alloys to be essentially single phase at room temperature or at high temperatures.
However, since the Co matrix undergoes an allotropic transformation from the low-temperature hexagonal closedpacked (hcp) structure to the high-temperature face-centred cubic (fcc) structure and showed a hysteresis, a two-phase matrix exists in Co-Re alloys at intermediate temperatures. Both phases (hcp and fcc) are not expected to be ordered (i.e., the alloying elements are randomly distributed within the particular phase). There is indication of ordering neither in the literature nor in the experimental data.

In pure $\mathrm{Co}$, the allotropic phase transformation occurs at around $417^{\circ} \mathrm{C}$ by a diffusionless martensitic mechanism [6] Alloying elements either increases or decreases the transformation temperature depending on whether they are hcp $(\mathrm{Cr}, \mathrm{Re})$ or fcc $(\mathrm{Ni}, \mathrm{Fe})$ stabilisers. In alloys containing $\mathrm{Ni}$, it is expected that the allotropic transformation temperature of the Co-Re-Cr alloys will be lowered. The overall phase evolution across the vast temperature range in the Ni-containing alloy was studied by in situ neutron diffraction (ND). A surprising split of the high-temperature Co fcc phase was found. This peak splitting was observed at 


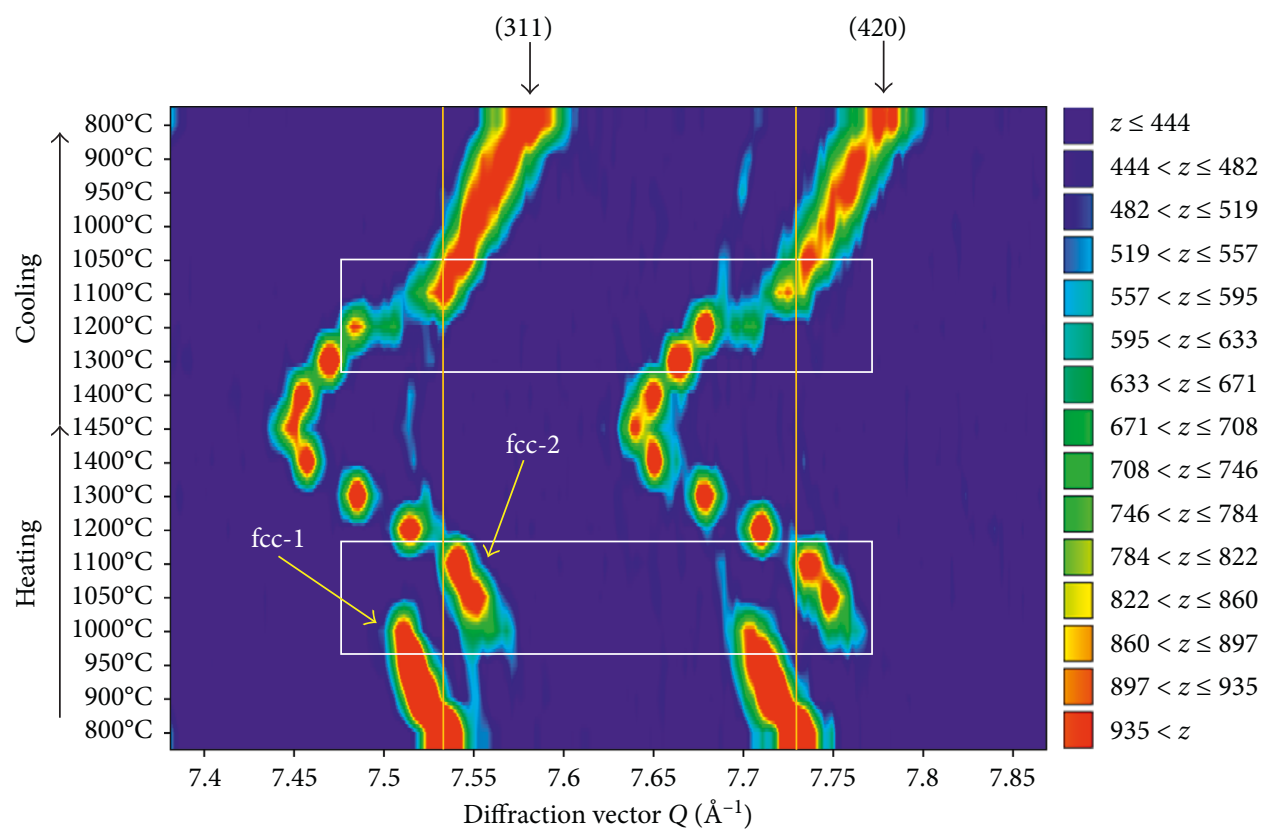

FIGURE 1: Intensity colour map of fcc reflections (331) and (420) as a function of temperature. Coexistence of two fcc phases during heating and cooling is highlighted. Low-intensity peaks crossing the whole temperature range (light blue) come from the $\mathrm{Al}_{2} \mathrm{O}_{3}$ sample holder parasitic reflections.

a temperature above which the $\sigma$-phase formation occurred. The neutron diffraction measurement also showed the coexistence of two cubic Co matrixes (both fcc) over a small temperature window. This has not been reported in any Co$\mathrm{Re}-\mathrm{Cr}$ alloys before which is a novel finding and was only made possible by the in situ measurement. The Co and Re-rich $\sigma$ phase (space group $P 4_{2} / m n m$ with $a \sim 9.03 \AA$ and $c \sim 4.67 \AA$ ) is a topologically closed-packed structure and-although it has been reported in other high-temperature alloys-it is generally avoided in those alloys as its presence causes brittleness. The sigma formation and the split in the Co fcc peak in Co-Re-Cr$\mathrm{Ni}$ alloys are therefore important discoveries for the Co-Re alloy development and was thus carefully investigated. The finding of the two cubic cobalt phases and the reason for their coexistence were studied in detail and reported in this article.

\section{Experimental}

Vacuum arc-melted Co-17Re-23Cr alloys with 8, 15, and 25\% $\mathrm{Ni}$ were solution heat treated at $1350^{\circ} \mathrm{C} / 5 \mathrm{~h}+1400^{\circ} \mathrm{C} / 5 \mathrm{~h}$ $+1450^{\circ} \mathrm{C} / 5 \mathrm{~h}$ and forced cooled in argon (designated as ST) prior to the in situ ND experiment. Cylindrical samples with $5 \mathrm{~mm}$ diameter and $4 \mathrm{~cm}$ height, equipped with the M6 thread to fix it through the $\mathrm{Al}_{2} \mathrm{O}_{3}$ holder inside the furnace, were prepared. In this paper, results only from $25 \% \mathrm{Ni}$ alloy are presented. Other compositions with $\mathrm{Ni}$ also show the similar evolution of the fcc splitting.

Structural and phase evolution was studied in situ by high-resolution neutron powder diffraction performed with the SPODI instrument at FRM II, Garching, Germany [7], equipped with a high-temperature furnace. Data were collected at room temperature (RT) before and after the in situ cycle and at 18 selected temperatures up to $1450^{\circ} \mathrm{C}$. The diffraction patterns at each temperature were collected in an angular range from 6 to $158^{\circ}$ of $2 \theta$ using a neutron wavelength of $0.1548 \mathrm{~nm}$. A hold time of about 40 minutes at each chosen temperature was needed to acquire a good diffractogram. The sample stick inside the furnace was rotationally oscillating around the vertical axis in the range of $\pm 45^{\circ}$ to average a possible texture contribution. The data were fitted by the Rietveld analysis using the Fullprof software [8].

Energy dispersive spectroscopy (EDS) and electron backscatter diffraction (EBSD) measurements were carried out along with electron microscopic imaging.

\section{Results and Discussions}

The refined diffraction pattern of the Co-17Re-23Cr alloy with $25 \% \mathrm{Ni}$ after ST heat treatment taken at RT reveals the following phase composition: $\sim 3 \% \mathrm{hcp}$ ( $\varepsilon \mathrm{Co}-\mathrm{P}_{3} / \mathrm{mmc}$ with $a \sim 2.56 \AA$ and $c \sim 4.15 \AA$ ) and $97 \%$ fcc ( $\gamma$ Co-Fm-3m with $a \sim 3.161 \AA$ ). This confirms that Ni is a fcc stabiliser in Co-Re$\mathrm{Cr}$ alloys. It has been reported that in pure Co about $30 \% \mathrm{Ni}$ is needed to stabilise the high-temperature fcc phase ( $\gamma \mathrm{Co}$ ) at RT [9].

During the heating, the first change starts at $900^{\circ} \mathrm{C}$ when the $\mathrm{Cr}_{2} \mathrm{Re}_{3}$-type $\sigma$ phase starts to form. With further heating, hcp phase disappears below $1000^{\circ} \mathrm{C}$ and the amount of $\sigma$ phase increases. In addition, an appearance of additional reflections is observed in the temperature interval 1000$1050^{\circ} \mathrm{C}$, and they are distinctly visible only at high diffraction angles. Figure 1 shows the evolution of the intensities of the cubic reflections (331) and (420) as a function of temperature, where the presence of both reflection sets is clearly visible. The new reflections match a phase with face-centred 


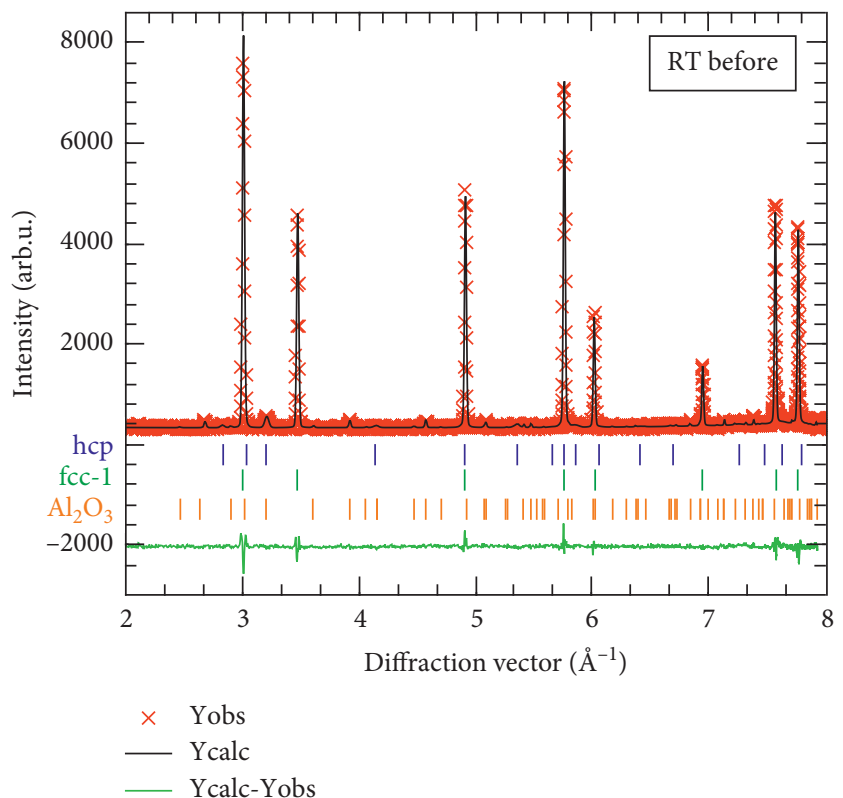

(a)

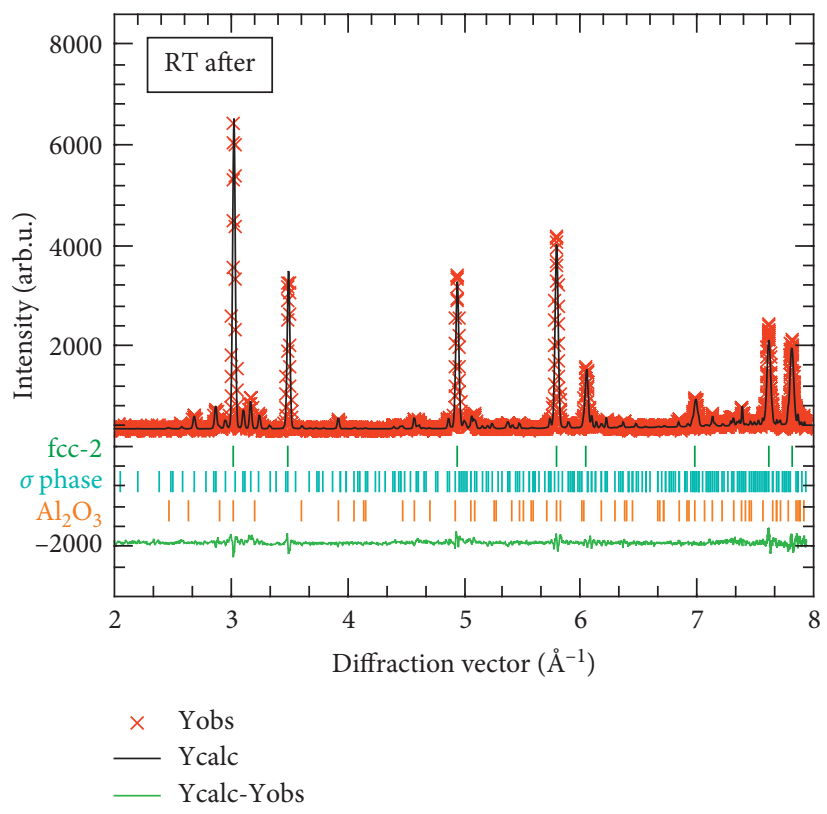

(b)

Figure 2: Measured and calculated diffraction patterns at room temperature before (a) and after (b) the thermal cycle. Bragg positions for individual phases are displayed as well.

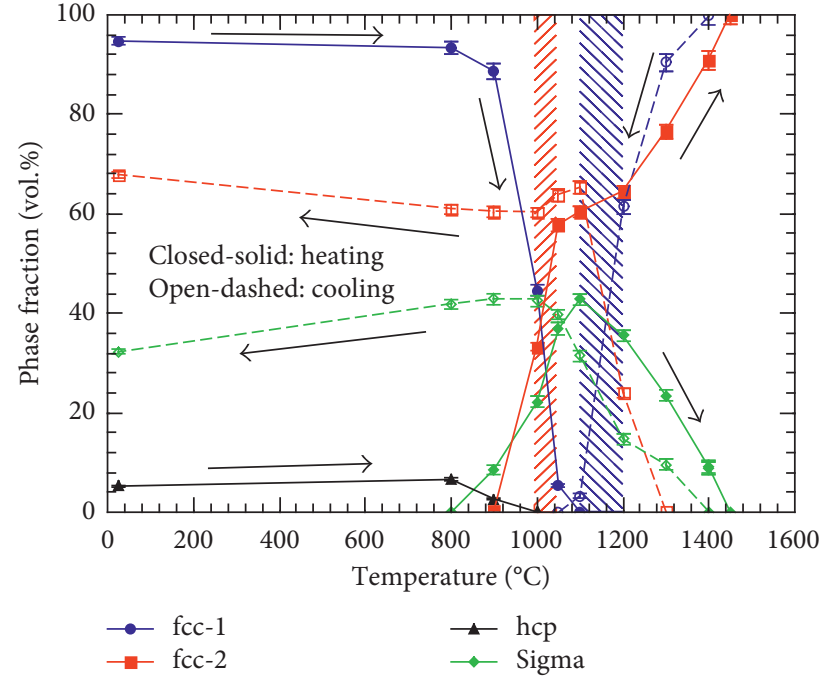

(a)

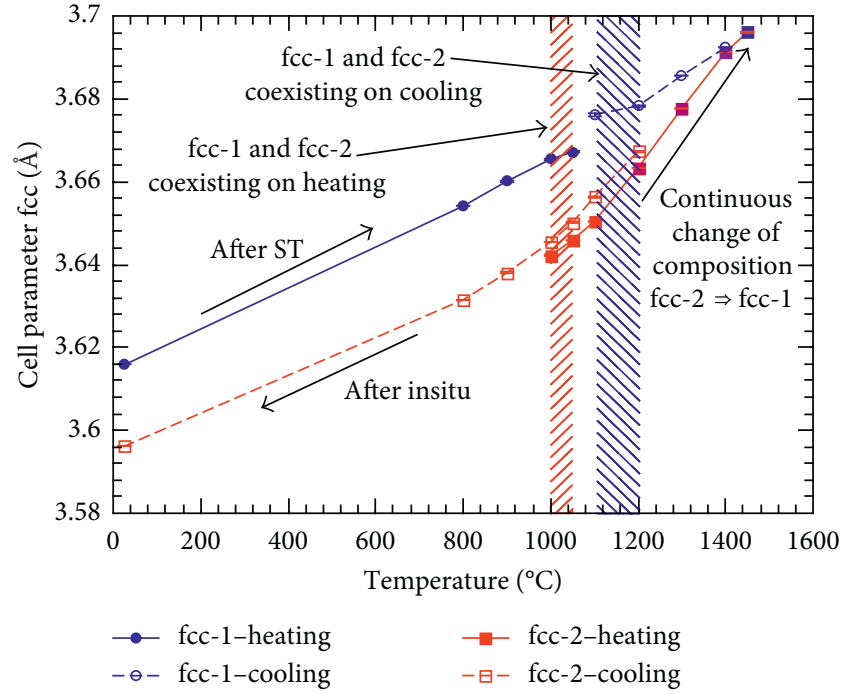

(b)

Figure 3: (a) Evolution of phase fraction for all phases as a function of temperature. (b) Evolution of the cell parameters for both fcc phases as a function of temperature during heating and cooling.

cubic symmetry (designated fcc-2) but with a smaller unit cell than the original fcc Co phase (designated fcc-1) already present in ST condition. The phase fraction of the fcc-1 decreases rapidly above $1000^{\circ} \mathrm{C}$, and the fcc-2 (together with $\sigma$ phase) are the only phases present above $1100^{\circ} \mathrm{C}$. Above $1100^{\circ} \mathrm{C}$, the amount of the $\sigma$ phase starts to decrease, and over $1400^{\circ} \mathrm{C}$ it is completely dissolved.

Figure 2 shows measured and calculated neutron diffraction patterns at room temperature before (Figure 2(a)) and after (Figure 2(b)) the thermal cycle. Parasitic reflections of $\mathrm{Al}_{2} \mathrm{O}_{3}$ coming from the sample holder were recognized and indexed in all patterns. The corundum phase was added to the structural refinements, as depicted in the figure, for proper phase fraction estimation.

In Figure 3(a), phase fractions of all phases are plotted as a function of temperature. During the $\sigma$ phase dissolution (above $1100^{\circ} \mathrm{C}$ ), the amount of fcc- 2 phase increases. Together with the volume fraction, also the cell parameter of fcc-2 phase increases (Figure 3(b)), indicating that the composition continuously changes as rhenium returns to the 


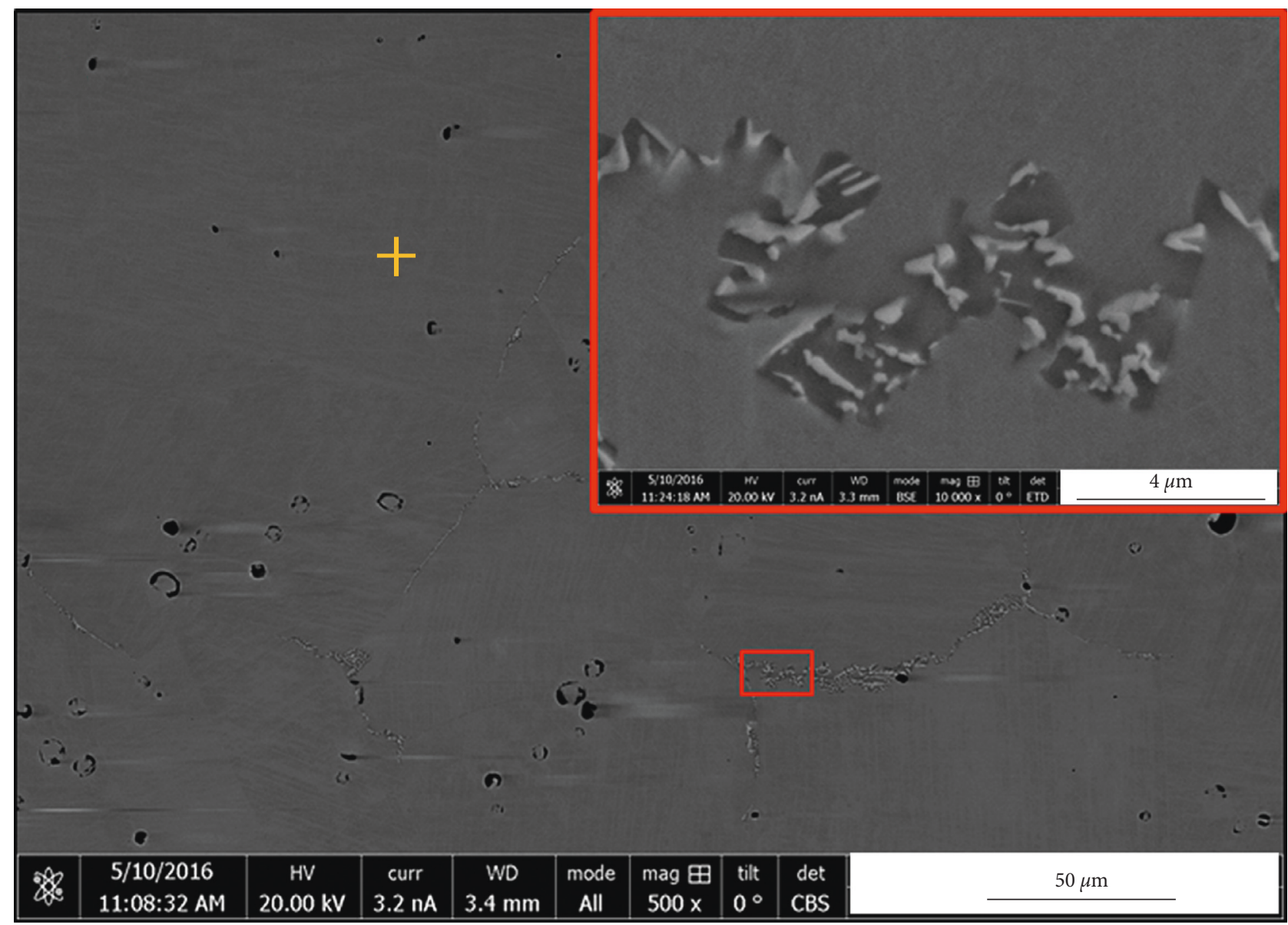

(a)
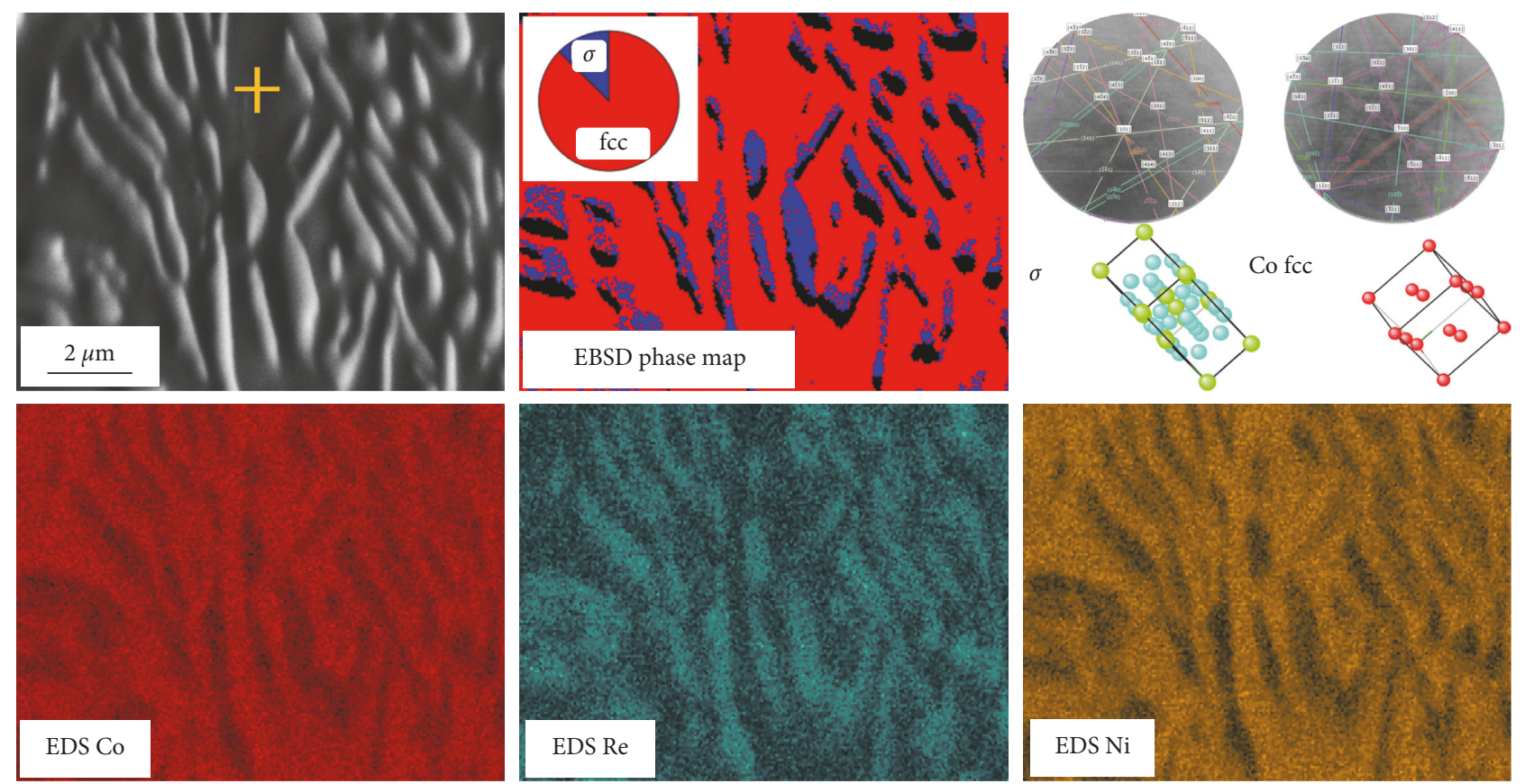

(b)

Figure 4: Micrograph of the Co-Re-23Cr-25Ni alloy showing the formation of the $\sigma$ phase (a) at grain boundaries in ST condition and (b) within grains after $800^{\circ} \mathrm{C}(100 \mathrm{~h})$ exposure. The EBSD phase map and EDS elemental distribution are also shown. Compositions were determined by EDS at cross marked positions in (a) and (b), and results are listed in Table 1. 
TABle 1: Composition of the fcc Co matrix measured on the Co17Re-23Cr-25Ni alloy by EDS at positions marked by + in Figure 4.

\begin{tabular}{lcccc}
\hline Heat treatment & $\mathrm{Co}$ & $\mathrm{Re}$ & $\mathrm{Cr}$ & $\mathrm{Ni}$ \\
\hline ST & 35.50 & 16.85 & 23.91 & 23.74 \\
ST +100 hours at $800^{\circ} \mathrm{C}$ & 38.22 & 12.18 & 21.97 & 27.63 \\
\hline
\end{tabular}

fcc matrix from the continuously dissolving $\sigma$ phase. At the temperature of $1450^{\circ} \mathrm{C}$, where all $\sigma$ phases are dissolved, the cell parameters of the cubic matrix again become the same as for the initial matrix (fcc-1), corrected for thermal expansion.

Coexistence of the two fcc phases and continuous composition change of Co matrix are linked with the formation/ dissolution of the $\sigma$ phase which is a Re-rich phase $\left(\mathrm{Cr}_{2} \mathrm{Re}_{3}\right.$ type). It has been reported that $\sigma$ phase formation occurs through a cellular reaction, starting at grain boundaries [10]. Since the cellular reaction is diffusion controlled, there is a partitioning of elements from the Co matrix to the $\sigma$ phase, and thereby rhenium and chromium are depleted in the matrix, particularly locally between the $\sigma$ particles. The matrix region with a lower concentration of rhenium (which has a bigger atomic radius in comparison to $\mathrm{Co}, \mathrm{Cr}$, and $\mathrm{Ni}$ ), is then expected to have a smaller unit cell. Since the atomic diffusion process takes place, $\sigma$ formation is strongly dependent on the rate of change of temperature over the interval where $\sigma$ is expected to nucleate. Due to forced cooling in argon, the formation of $\sigma$ phase is seldom observed after the ST procedure. However, during holding at the temperature or when heating/cooling slowly, rhenium diffusion aids $\sigma$ phase formation. During heating, $\sigma$ phase is formed by partitioning of rhenium from the matrix. The rhenium-depleted matrix is the fcc-2 phase with smaller cell parameters. The cellular reaction starts at some grain boundaries, and the reaction front moves into grains through diffusion, and during the small temperature window of $1000^{\circ} \mathrm{C}-1050^{\circ} \mathrm{C}$, the two fcc phases (fcc-1 and fcc-2) coexist across the reaction front. At $1100^{\circ} \mathrm{C}$, all grains are affected by the cellular reaction, and the maximum amount (40\%) of the $\sigma$ phase is reached; thus, only one fcc matrix (fcc-2) remains. On further heating, $\sigma$ phase, which is unstable at higher temperatures $\left(>1200^{\circ}\right)$, starts to dissolve, and rhenium is again partitioned back to the Co matrix. Therefore, the unit cell size of fcc-2 gradually increases (Figure 3(b)), and when all of $\sigma$ is dissolved at $1400^{\circ} \mathrm{C}$, the fcc matrix has the same composition as the original starting matrix composition after ST, and thus the same lattice parameter as fcc- 1 corrected for the thermal expansion.

The typical image of the secondary $\sigma$ phase formation is shown in Figure 4(a). Figure 4(b) shows the microstructure at RT after 100 hours of hold time at $800^{\circ} \mathrm{C}$. The phases are identified by EBSD, and the element distributions are mapped by EDS. The matrix compositions at the points marked by the + symbol in the two images, quantitatively measured by EDS, are shown in Table 1 . The $\sigma$ phase is enriched in rhenium, and the matrix in the region between the $\sigma$ precipitates is depleted in rhenium (Figure 4(b) and Table 1). EBSD confirms the matrix in this region is fcc Co (Figure 4(b)).
During cooling, $\sigma$ phase starts to form above $1300^{\circ} \mathrm{C}$ in the matrix which has the nominal composition (fcc-1). At $1200^{\circ} \mathrm{C}$, when $\sigma$ phase fraction is about $16 \%$, the secondary fcc phase with smaller cell parameter (fcc-2) is detected (Figure 3). The amount of fcc-1 rapidly decreases when $\sigma$ phase volume increases. The temperature interval when two fcc phases coexist during cooling (also marked in Figure 1) is shifted towards higher values $\left(1200-1100^{\circ} \mathrm{C}\right)$. Below $1100^{\circ} \mathrm{C}$, only $\sigma$ phase and matrix with the smaller unit cell (fcc-2) are present what persists till RT. Formation of hcp phase during cooling was not observed in the in situ experiment. Composition at RT after cooling is $33 \% \sigma$ phase and $67 \% \mathrm{fcc}$ phase.

\section{Conclusions}

Phase evolution of the Co-17Re-23Cr-25Ni alloy was studied in situ by neutron powder diffraction at high temperatures. An appearance of additional fcc phase with a smaller unit cell within the original fcc matrix was observed during heating and cooling. Such fcc matrix phase splitting has not yet been reported in Co-Re alloys. The presence of two coexisting fcc phases is related to the phenomenon of formation and dissolution of the $\sigma$ phase. The $\sigma$ phase formed by cellular transformation involving diffusion of rhenium leads to rhenium-depleted regions within the matrix. On cooling to RT, the hcp Co phase did not form due to the slower cooling rates employed during in situ measurement compared to the ST heat treatment and due to the significant fcc stabilisation by means of $25 \% \mathrm{Ni}$.

\section{Data Availability}

The raw neutron diffraction data are available at MLZ Garching where the measurement was carried out. They are also available from the first author on request.

\section{Conflicts of Interest}

There are no conflicts of interest.

\section{Acknowledgments}

The authors thank the Heinz Maier-Leibnitz Zentrum in Garching, Germany, for providing beamtime. This work was supported by the German Research Foundation (DFG) (Grant no. RO 2045/32-1) at TU Braunschweig and by the Czech Science Foundation (Grant no. 14-36566G).

\section{References}

[1] D. Mukherji, J. Rösler, J. Wehrs et al., "Application of in situ neutron and X-ray measurements at high temperatures in the development of Co-Re-based alloys for gas turbines," Metallurgical and Materials Transactions A, vol. 44, no. 1, pp. 22-30, 2013.

[2] J. Rösler, D. Mukherji, and T. Baranski, "Co-Re-based alloys: a new class of high-temperature materials?," Advanced Engineering Materials, vol. 9, no. 10, pp. 876-881, 2007.

[3] T. Depka, "Co-Re-based Alloys - A First Assessment of Creep Properties and Microstructural Evolution at High Temperature," 
Ph.D. thesis, Ruhr Universität Bochum, Bochum, Germany, 2012.

[4] D. Mukherji, M. Klauke, P. Strunz et al., "High-temperature stability of Cr-carbides in an experimental Co-Re-based alloy," International Journal of Materials Research, vol. 101, no. 3, pp. 340-348, 2010.

[5] J. W. Christian, "A theory of the transformation in pure cobalt," Proceedings of the Royal Society A: Mathematical, Physical and Engineering Sciences, vol. 206, no. 1084, pp. 5164, 1951.

[6] D. Mukherji, P. Strunz, R. Gilles, M. Hofmann, F. Schmitz, and J. Rösler, "Investigation of phase transformations by insitu neutron diffraction in a Co-Re-based high-temperature alloy," Materials Letters, vol. 64, no. 23, pp. 2608-2611, 2010.

[7] M. Hoelzel, A. Senyshyn, N. Juenke, H. Boysen, W. Schmahl, and H. Fuess, "High-resolution neutron powder diffractometer SPODI at research reactor FRM II," Nuclear Instruments and Methods in Physics Research Section A: Accelerators, Spectrometers, Detectors and Associated Equipment, vol. 667, pp. 32-37, 2012.

[8] J. Rodríguez-Carvajal, "Recent advances in magnetic structure determination by neutron powder diffraction," Physica B: Condensed Matter, vol. 192, no. 1-2, pp. 55-69, 1993.

[9] Z. Nishiyama, Martensitic Transformation, Academic Press, New York, NY, USA, 1978.

[10] C.-C. Hsieh and W. Wu, "Overview of intermetallic sigma $(\sigma)$ phase precipitation in stainless steels," ISRN Metallurgy, vol. 2012, Article ID 732471, 16 pages, 2012. 


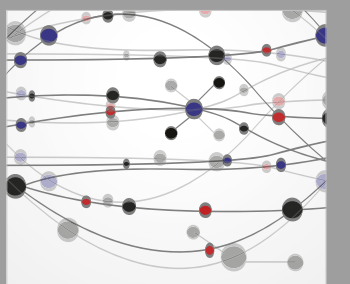

The Scientific World Journal
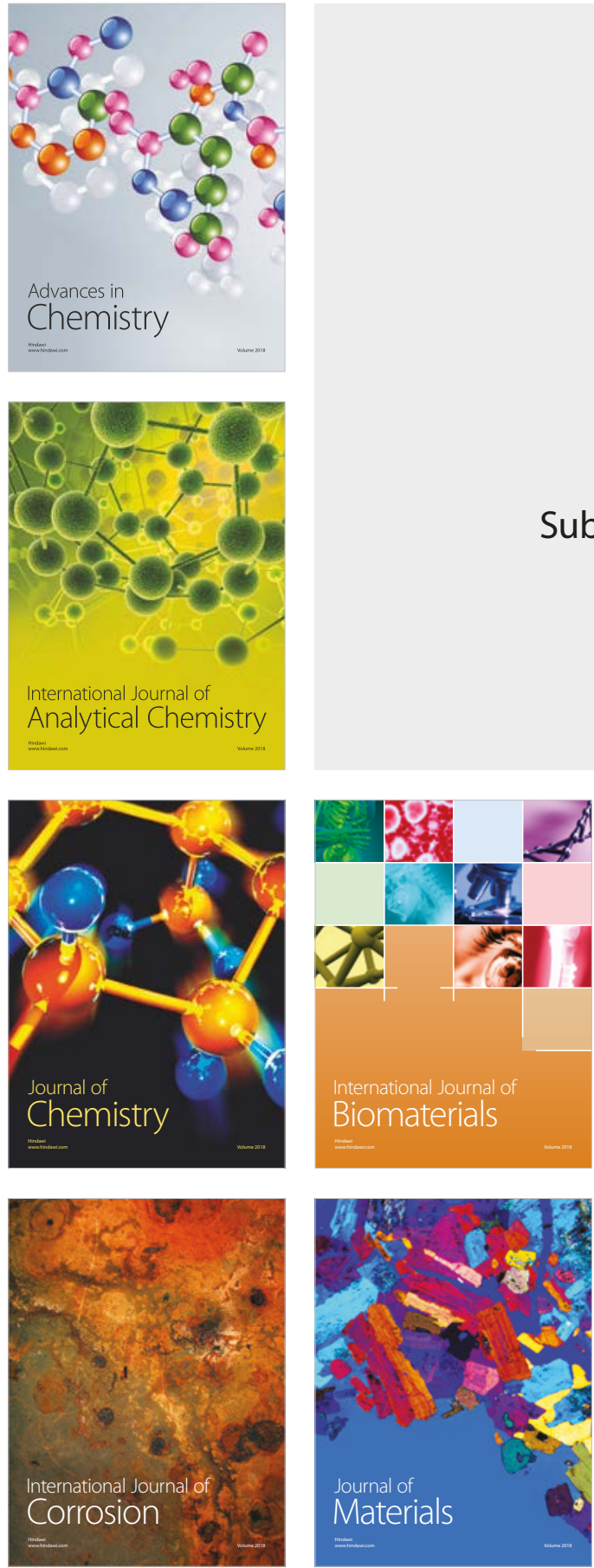

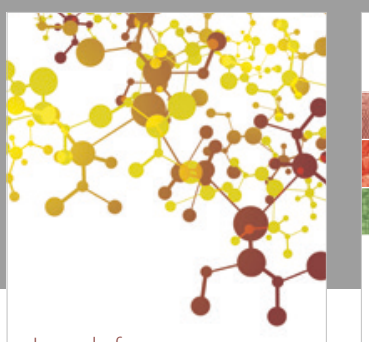

Journal of

Applied Chemistry
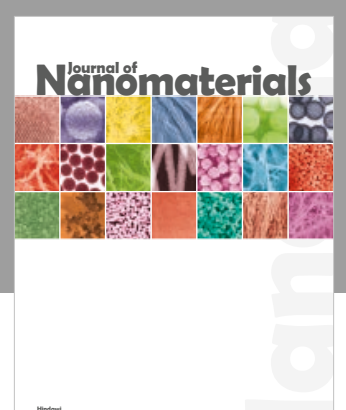

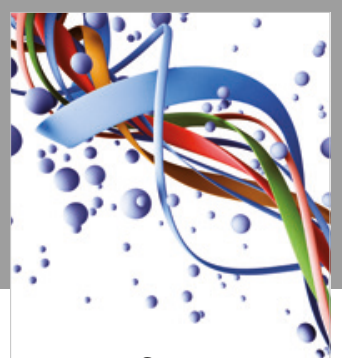

Scientifica

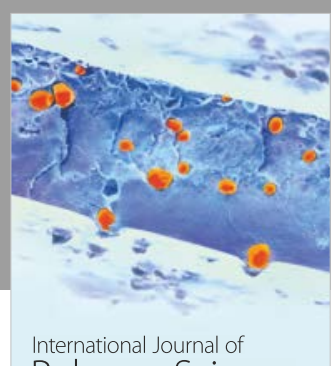

Polymer Science

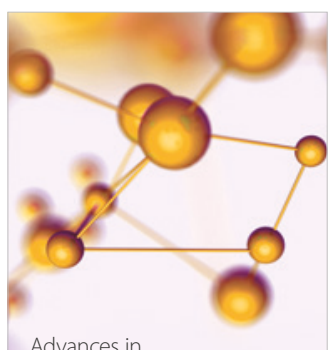

Physical Chemistry
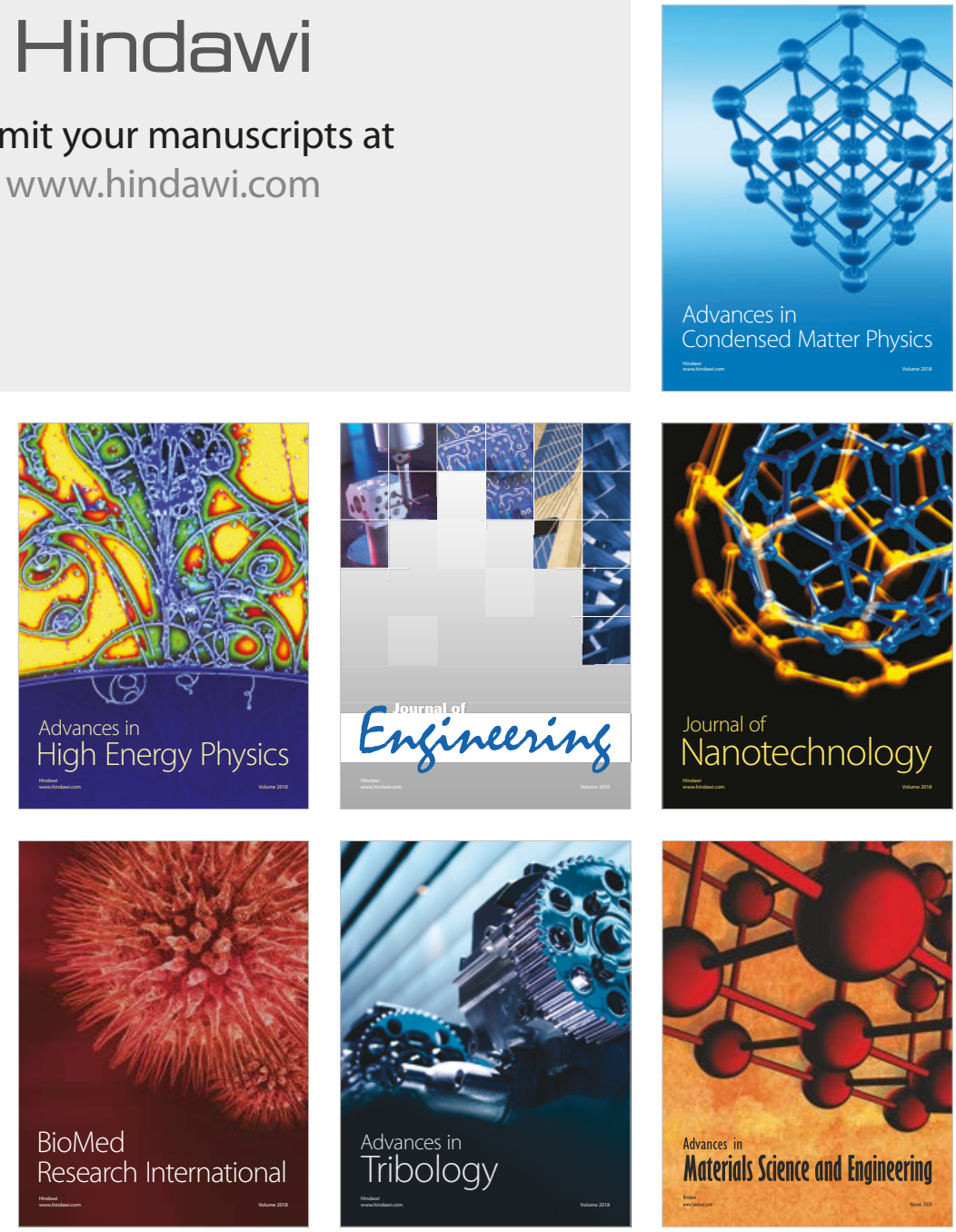